\title{
Tuberculous Pericardial Effusion in an Infant: A Case Report
}

\author{
Abhilasha Singh Panwar R. ${ }^{1}$, Rewat Meshram², Amar Taksande ${ }^{3}$ \\ 1, 2, 3 Department of Paediatrics, Jawaharlal Nehru Medical College, Sawangi, Wardha, Maharashtra, India.
}

\section{INTRODUCTION}

Tuberculous pericardial effusion is a rare manifestation of extra pulmonary tuberculosis. In children, $25 \%$ of the cases of tuberculosis are extra pulmonary tuberculosis. Echocardiography is an accurate bedside investigation for diagnosis. Pericardiocentesis with antituberculous therapy is the mainstay of treatment. This is a rare case report of an infant with TB pericardial effusion.

Pericardial effusion is the accumulation of fluid in the pericardial sac. ${ }^{1}$ Worldwide it occurs most commonly due to malignancy, tuberculosis and various viral, immunological and idiopathic reasons. ${ }^{2}$ In tuberculosis endemic countries, Mycobacterium tuberculosis is a frequent cause of pericardial effusion. ${ }^{3}$ Approximately, four percent of the cases of acute pericarditis and $10 \%$ of cases of cardiac tamponade is attributed to tuberculosis. ${ }^{4}$ It is usually seen in adults. Here we are presenting a case of tuberculous pericardial effusion in an infant.

\section{PRESENTATION OF CASE}

A 9-month-old male child born out of a non-consanguineous marriage was brought with complaints of fever since 3 days. Fever was high grade, intermittent, with no diurnal variation and was not relieved on taking medications. Next day he developed dry cough along with increased difficulty in breathing. There is no history of contact with a case of tuberculosis. He was born full term via normal vaginal delivery to a primigravida mother. He is a developmentally normal child who had received BCG vaccination at birth and has been vaccinated appropriately for his age. Previously he had been admitted for cough and cold at 7 months of age during which time he was on oxygen and received antibiotics. On admission, patient was febrile, with tachypnoea and tachycardia. His heart rate: 160 / min, respiratory rate: 48 / min, blood pressure: $90 / 60 \mathrm{mmHg}$ in the right upper limb. On cardiovascular examination, first and second heart sound were faintly heard and muffled. Respiratory examination revealed bilateral crepitation. Per abdomen examination was normal. No neurological deficit on central nervous system examination.

Haemoglobin - $9.2 \mathrm{gm} / \mathrm{dL}$, total count - 18,800 cells $/ \mathrm{mm}^{3}$ and platelet - 1.71 lakhs / $\mathrm{mm}^{3}$. C-reactive protein of 27 . Chest $\mathrm{x}$-ray was suggestive of cardiomegaly (cardiothoracic ratio $>0.6$ ). Two-dimensional echocardiography showed moderate amount of pericardial effusion with fibrinous strands suggestive of TB pericardial effusion. Pericardiocentesis was done and $35 \mathrm{~mL}$ of pericardial fluid was drained. Pericardial fluid glucose: $90 \mathrm{mg}$ / dL, proteins: 46 gm / dL, LDH: 549 IU / L, albumin: $2.3 \mathrm{gm} / \mathrm{dL}$, Total leucocyte count - 272 cells / uL. Differential leucocyte count: P: 20 \%, L: 80 \%, Adenosine deaminase activity: 28.8 U / L. Pericardial fluid gene expert (cartridge based nucleic acid amplification test) was positive for Mycobacterium tuberculosis. Patient was started on antitubercular drugs for 6 months. He was given steroids for 15 days. On follow up examination patient had weight gain, no other complaints and repeat 2D echo was normal.
Corresponding Author: Dr. Abhilasha Singh Panwar R, Department of Paediatrics, Acharya Vinoba Bhave Rural Hospital, Sawangi Meghe, Wardha -442001,

Maharashtra, India.

E-mail: abhilasha892@gmail.com

DOI: $10.14260 / j e m d s / 2020 / 714$

How to Cite This Article:

Panwar RAS, Meshram R, Taksande A. Tuberculous pericardial effusion in an infant: a case report. J Evolution Med Dent Sci 2020;9(43):3256-3257, DOI: $10.14260 / \mathrm{jemds} / 2020 / 714$

Submission 15-07-2020,

Peer Review 16-09-2020,

Acceptance 23-09-2020,

Published 26-10-2020.

Copyright (C) 2020 Abhilasha Singh Panwar $R$. et al. This is an open access article distributed under Creative Commons Attribution License [Attribution 4.0 International (CC BY 4.0)] 


\section{DISCUSSION}

Pericardial effusion is a rare presentation of extra pulmonary tuberculosis. ${ }^{5}$ In the study conducted by Bagri et $\mathrm{al}^{6}$ it was found that tuberculosis was the most common cause of pericardial effusion which was seen in $52 \%$ of the cases. In a study conducted by Peter et al 7 it was found that pericardial effusion of infectious cause (58.8\%) was the most common, with TB (70 \%) being the most prevailing infectious cause. TB pericardial effusion usually presents as fever which is gradually increasing. ${ }^{8}$

Due to reverse lymphatic flow of the bacteria from peritracheal, peribronchial, or mediastinal lymph node or by haematogenous spread from primary tuberculous infection will lead to involvement of the heart. ${ }^{9}$ TB pericarditis occurs due to delayed hypersensitivity induced by the activation of lymphocytes releasing lymphocytokines that activate macrophages, leading to granuloma formation and pericardial effusion. ${ }^{3}$ It commences insidiously and has constitutional symptoms like weight loss and lassitude. The patient usually has fever, dyspnoea; while precordial pain is seen less often. Pericardial rub raised jugular venous pressure and pulsus paradoxus is present. Peripheral lymphadenopathy with matting may be present. ${ }^{8}$ Beck's triad involves muffled heart sounds, hypotension and distended neck veins. In case of cardiac tamponade, patient presents with shock. ${ }^{10}$ Initially in tuberculous pericarditis there is fibrinous exudation due to immune response in stage one which becomes serosanguinous in second stage.

There is pericardial thickening with granulomatous caseation in stage three followed by scarring and constriction in the fourth stage. ${ }^{5}$ It is difficult to diagnose TB pericardial effusion as only $2 \%$ smear positive cases of pericardial fluid are there and only $38-56 \%$ of culture positivity is present. Additional test for tuberculosis is adenosine deaminase levels in pericardial fluid..$^{9}$ Johari et $\mathrm{a}^{5}$ showed that $2 \mathrm{D}$ echo showed formation of fibrin, septations and granulomas with adherence and thickening of leaflets. ${ }^{5}$ Early identification and diagnosis of pericardial effusion is necessary to decrease the mortality rates. It is approached aggressively by pericardial fluid tapping and starting of antitubercular therapy. ${ }^{5}$ Wallrauch et.al ${ }^{11}$ said that it was necessary to drain the pericardial fluid to relieve the signs of haemodynamic instability thus causing resolution of the tamponade. Patients are to be started with a six-month course of anti-TB medication, with a combination of isoniazid, rifampicin, ethambutol and pyrazinamide for two months, subsequently isoniazid and rifampicin for four months. This regime of treatment has been shown to be highly effective based on a few previous trials. ${ }^{5}$ In a review by Schutz et al. ${ }^{12}$ after starting steroids the rate of constrictive pericarditis decreased by $44 \%$. In high prevalence areas it is better to initiate empiric antituberculous therapy in the presence of large pericardial effusion as there is lack of proper diagnostic methods. ${ }^{9}$

\section{CONCLUSIONS}

Diagnosing TB pericardial effusion is challenging especially in an endemic area. Once diagnosed it has to be managed aggressively with pericardiocentesis and anti-tubercular therapy.

Financial or other competing interests: None.

Disclosure forms provided by the authors are available with the full text of this article at jemds.com.

\section{REFERENCES}

[1] Willner DA, Goyal A, Grigorova Y, et al. Pericardial effusion. StatPearls Treasure Island (FL): StatPearls Publishing 2020: p. 2.

[2] Singh Y, Ahmad S, Srivastava S. Trends in etiology and inhospital outcome of patients with pericardial effusion. JIACM 2008;9(2):96-9.

[3] Jung IY, Song YG, Choi JY, et al. Predictive factors for unfavorable outcomes of tuberculous pericarditis in human immunodeficiency virus-uninfected patients in an intermediate tuberculosis burden country. BMC Infect Dis 2016;16(1):719.

[4] Wanjari K, Baradkar V, Mathur M, et al. A case of tuberculous pericardial effusion. Indian J Med Microbiol 2009;27(1):75-7.

[5] Johari MI, Ramli AW, Mat Lawi FM, et al. A rare case of purulent pericardial TB. Cureus 2019;11(8):e5356.

[6] Bagri NK, Yadav DK, Agarwal S, et al. Pericardial effusion in children: experience from tertiary care center in northern India. Indian Pediatr 2014;51(3):211-3.

[7] Peter ID, Asani MO, Ibrahim Aliyu I. Pericardial effusion and outcome in children at a Tertiary Hospital in NorthWestern Nigeria: a 2-year retrospective review. Res Cardiovasc Med 2019:8(1):14-8.

[8] Cherian G. Diagnosis of tuberculous aetiology in pericardial effusions. Postgrad Med J 2004;80(943):2626.

[9] Mayosi BM, Wiysonge CS, Ntsekhe M, et al. Clinical characteristics and initial management of patients with tuberculous pericarditis in the HIV era: the investigation of the management of pericarditis in Africa (IMPI Africa) registry. BMC Infect Dis 2006;6:2.

[10] Guven H, Bakiler AR, Ulger Z, et al. Evaluation of children with a large pericardial effusion and cardiac tamponade. Acta Cardiol 2007;62(2):129-33.

[11] Heller T, Wallrauch C, Lessells RJ. Tuberculosis pericarditis with cardiac tamponade: management in the resource-limited setting. Am J Trop Med Hyg 2010;83(6):1311-4.

[12] Schutz C, Davis AG, Sossen B, et al. Corticosteroids as an adjunct to tuberculosis therapy. Expert Rev Respir Med 2018;12(10):881-91. 